\title{
The Age of the Universe Predicted by a Time-Varying $G$ Model?
}

\author{
Christopher Pilot \\ Physics Department, Gonzaga University, Spokane, WA, USA \\ Email: pilot@gonzaga.edu
}

How to cite this paper: Pilot, C. (2019) The Age of the Universe Predicted by a Time-Varying $G$ Model? Journal of High Energy Physics, Gravitation and Cosmology, 5, 928-934.

https://doi.org/10.4236/jhepgc.2019.53048

Received: June 18, 2019

Accepted: July 28, 2019

Published: July 31, 2019

Copyright () 2019 by author(s) and Scientific Research Publishing Inc. This work is licensed under the Creative Commons Attribution International License (CC BY 4.0).

http://creativecommons.org/licenses/by/4.0/

\begin{abstract}
Based on previous work, it is shown how a time varying gravitational constant can account for the apparent tension between Hubble's constant and a newly predicted age of the universe. The rate of expansion, about nine percent greater than previously estimated, can be accommodated by two specific models, treating the gravitational constant as an order parameter. The deviations from $\Lambda \mathrm{CDM}$ are slight except in the very early universe, and the two time varying parametrizations for $G$ lead to precisely the standard cosmological model in the limit where, $\dot{G} / G \rightarrow 0$, as well as offering a possible explanation for the observed tension. It is estimated that in the current epoch, $\dot{G} / G=-0.06 H_{0}$, where $H_{0}$ is Hubble's parameter, a value within current observational bounds.
\end{abstract}

\section{Keywords}

Universe, Gravitational Constant, Hubble's Constant, Standard Cosmological Model

Recently, it has been observed that the universe is much less old than previously thought [1] [2] [3]. The latest galaxy studies indicate that the rate of expansion is about 9 percent faster than previous estimates. Instead of being 13.8 billion years old, current estimates would indicate the age of the universe at a more modest age of, 12.5 - 13 Gyr. As stated by members of the research team concerning the latest findings, "there becomes a very strong likelihood that we're missing something in the cosmological model". As indicated in the title of the work, there now is "stronger evidence for physics beyond $\Lambda \mathrm{CDM}$ ".

In this short note, we would like to bring to the attention of the reader that a cosmologically time-varying $G$ can accommodate such a result. As a particular 
example, we refer the reader to reference [4]. In particular, see Figure/Graph 6a, in Appendix D, in that reference, which we reproduce here as Figure 1. This figure replicates exactly the newest age without any modifications or revisions of existing cosmological parameters as determined by the Planck XIII collaboration [5]. Figure 1 gives the "look back" times for three specific models, the $\Lambda \mathrm{CDM}$ model, model A, and, model B, as a function of the cosmic scale parameter, " $a$ ". Models, A, and B, are two specific time-varying $G$ models. Specifically this figure graphs the age correction factors, $F$, for the various models under consideration. The specific values, as determined in reference [4], are $F=(0.9559,0.8688,0.9018)$ for models, $(\Lambda \mathrm{CDM}$, model A, model B), respectively. We assumed $\Omega_{R A D}=0.000083, \Omega_{\text {MATTER }}=0.3089$ and $\Omega_{\Lambda}=0.6911$ as our input values, as determined by the Planck XIII collaboration. With these values for the age correction factor and a Hubble value equal to,

$H_{0}=67.74 \mathrm{~km} /(\mathrm{s} \cdot \mathrm{Mpc})$, we obtain 13.8 Gyr for the $\Lambda \mathrm{CDM}$ model. But for models $\mathrm{A}$, and $\mathrm{B}$, we obtain, respectively, $12.5 \mathrm{Gyr}$, and, $13.0 \mathrm{Gyr}$, as the age of the universe without any further modifications. These are a precise match with what is being claimed in reference [1].

A time-varying gravitational constant is, of course, an old idea, going back to the work of Dirac in his large number hypothesis (LNH). See references, [6]-[12]. Dirac's original proposal was that quantum physics is related to cosmic evolution via the gravitational constant. In fact, it was suggested that, $\dot{G} / G \sim-H$, where $G$ is Newton's constant, $H$ is Hubble's parameter, and $\dot{G}$ is the time-varying gravitational constant. Observational data rule out a time varying $G$ of this order of magnitude. See reference [4] for many references indicating this fact. However, we claimed that, $\dot{G} / G \sim-0.06 H$, a value within current observational bounds. This value was derived in order to explain the quintessence parameter. Moreover, as noted in my original work, and as recognized by various other authors [13], it has been suggested that the vacuum energy formed in the inflationary period may provide a direct link between atomic physics and cosmology. Quantum effects may still dictate the ultimate fate of the universe via the cosmological constant, as well as its previous evolution. This is precisely what I attempted to show in my previous work. I suggested a model which could hypothetically explain the cosmological constant problem, both past and

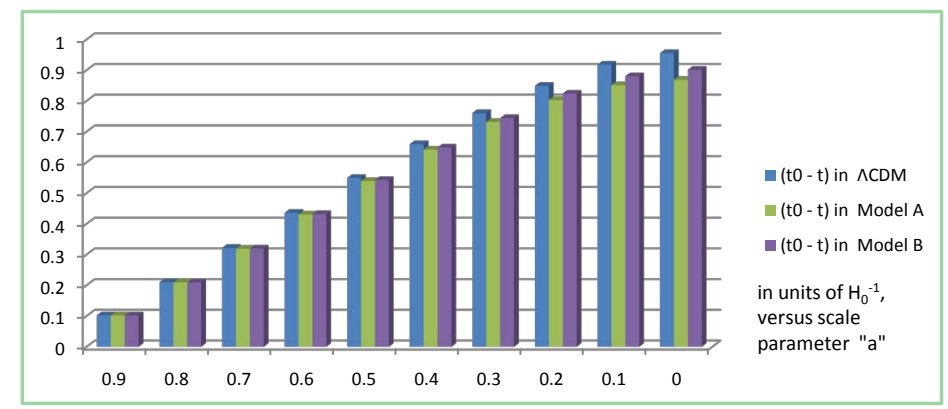

Figure 1. Age Correction Factors for Three Models, $\Lambda \mathrm{CDM}$, model A, and model B. $H_{0}$ is Hubble's constant. 
present, using a time varying $G$ model. We believe that we also derived a cosmic evolution with this time-varying gravitational constant which led to sensible results at key cosmological epochs.

The two models, $A$ and $B$, were introduced in reference [4] in order to explain the quintessence parameter, $w=-0.98$, versus $w=-1$, which holds in the $\Lambda \mathrm{CDM}$ model. We also sought to explain the cosmological constant fine tuning problem, the discrepancy between the present day very weak value of the cosmological constant, and the much greater vacuum energy found in earlier epochs. We assumed a connection exists, and that the cosmological constant is a characteristic of the vacuum. The present-day observed value is, $\Lambda_{O B S}=1.11 \times 10^{-52} \mathrm{~m}^{-2}=4.33 \times 10^{-66}(\mathrm{eV})^{2}$. At the very beginning of cosmic evolution, $\Lambda_{V A C U U M}=(\text { Planck Length })^{-2}=3.83 \times 10^{69} \mathrm{~m}^{-2}=1.22 \times 10^{28}(\mathrm{eV})^{2}$. This discrepancy in length squared, amounting to 121 orders of magnitude, between $\Lambda_{V A C U U M}$ and, $\Lambda_{O B S}$, has often been referred to as the "worst fine-tuning problem" in physics. To explain both the cosmological fine tuning problem, as well as the observationally determined quintessence parameter, $w=-0.98$, we assumed that the inverse gravitational constant, $G^{-1}$, is in reality, an order parameter varying very slowly at present with respect to cosmological time.

In order to derive, $w=-0.98$, we parametrized $G^{-1}=G^{-1}(T)=G^{-1}(a)$, by means of two separate functions, which we distinguished as model $A$, and model $B$. The cosmic scale parameter, " $a$ " is related to CMB temperature, $T$, by $a=(2.725 / T)=(1+z)^{-1}$, where $z$ is the redshift. In model A, we set

$$
G^{-1}=G_{\infty}^{-1}\left(1-\mathrm{e}^{-x}\right)
$$

In Equation, (1), " $x$ " is defined as $x \equiv b / T=a b / T_{0} \quad$ where " $b$ " is a constant to be determined, having units of degrees Kelvin, $T_{0}=2.275 \mathrm{~K}$, and " $a$ " is our scale parameter. In the present epoch, “ $a$ " $=1$, and thus, $x_{0} \equiv b / T_{0}$. In Equation (1) $G_{\infty}^{-1}$ is the saturation value of $G^{-1}$, applicable in the limit where the CMB temperature approaches zero, or equivalently, when " $a$ " approaches infinity.

In model $\mathrm{B}$, we have correspondingly,

$$
G^{-1}=G_{\infty}^{-1} L(x) \equiv G_{\infty}^{-1}[\operatorname{coth}(x)-1 / x]
$$

In Equation, (2), $L(x)$ is the Langevin function, defined by the equation $L(x) \equiv[\operatorname{coth}(x)-1 / x]$. As before $x \equiv b / T=a b / T_{0}$ where " $b$ " is a constant to be determined, having units of degrees Kelvin, and, $x_{0} \equiv b / T_{0}$. In Equation (2), $G_{\infty}^{-1}$ is a different saturation value for $G^{-1}$, but defined in the same way. In the limit where $T$ approaches zero, $G^{-1}$ approaches $G_{\infty}^{-1}$. Both models, A, and B, gave a current time variation of, $\dot{G} / G=-0.06 H_{0}$, where $H_{0}$ is Hubble's parameter. This variance in $G$ value is within current observational bounds. To satisfy, $w=-0.98$, we found that,

$$
\begin{aligned}
& x_{0}=4.28, b=11.663 \mathrm{~K} \\
& x_{0}=17.67, b=48.15 \mathrm{~K}
\end{aligned}
$$

Thus, for any scale parameter, “a”, Equations (1) and (2), can be utilized to 
find the corresponding $G^{-1}=G^{-1}(a)$ value at any cosmological epoch. A graph of both functions as a function of cosmic scale parameter is presented in Figure 2 .

Moreover, it was argued that $G^{-1}$ surfaced at a temperature of

$$
\begin{gathered}
T_{C}=6.20 \times 10^{21} \mathrm{~K} \\
T_{C}=7.01 \times 10^{21} \mathrm{~K}
\end{gathered}
$$

Beyond this temperature, gravity, as we currently know it, did not exist. Therefore gravity is considered to be an emergent "low energy" phenomenon, which came into being at very high temperatures. We also claimed that, if $G$ is not fundamental, then there is nothing fundamental about the Planck scale. It is to be noted that even though models, A, and B, are underpinned by two different functions, the inception of gravity is roughly at the same temperature. We consider this to be more than a coincidence. The temperatures indicated by Equations, (5), and, (6), are well below the Planck temperature of, $1.42 \times 10^{32} \mathrm{~K}$, but well above the temperature of $10^{16} \mathrm{~K} \approx 1 \mathrm{TeV}$, where all particles in the standard model are ultra-relativistic [14] [15].

Models, A, and B, lead to saturation values of

$$
\begin{aligned}
& \left.\left(G_{\infty}^{-1}\right)\right|_{A}=1.014 G_{0}^{-1} \quad(\text { model A; large “" } \geq 2)(7) \\
& \left.\left(G_{\infty}^{-1}\right)\right|_{B}=1.054 G_{0}^{-1} \quad(\text { model B; large “" } a 10)(8)
\end{aligned}
$$

This means that, $G_{0}$, the current value of Newton's constant will decrease further, until

$$
\begin{aligned}
& \left.\left(G_{\infty}\right)\right|_{A}=0.986 G_{0} \quad(\text { model A; large “" } a 2)(9) \\
& \left.\left(G_{\infty}\right)\right|_{B}=0.949 G_{0} \quad(\text { model B; large “ } a " \geq 10)(10)
\end{aligned}
$$

The details are presented in reference [4].

With these two time varying $G$ models, we can make a case for the quintessence parameter, $w=-0.98$. We can also show that the cosmological constant, $\Lambda$, scales. In fact,

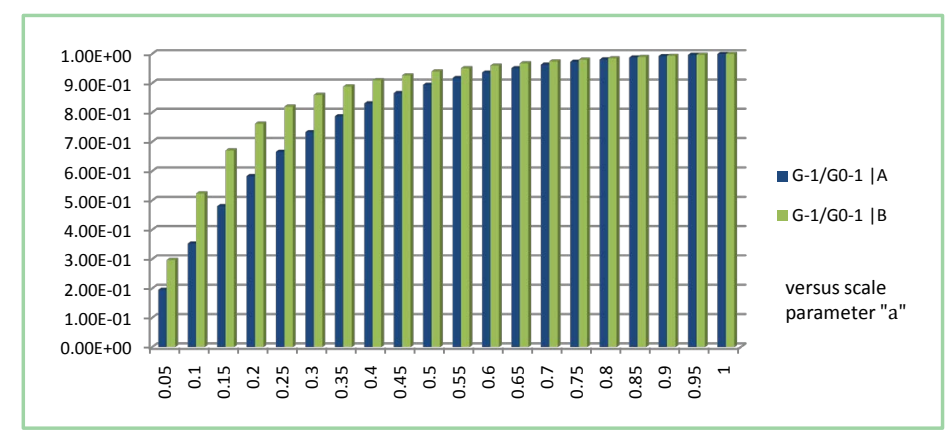

Figure 2. Order Parameters $G^{-1} / G_{0}^{-1}$ plotted as a Function of Cosmic Scale Parameter, "a", for models A, and B. $G_{0}$ is Newton's constant in the current epoch. 


$$
G / G_{0}=\rho_{\Lambda} / \rho_{\Lambda_{0}}=\left(\Lambda / \Lambda_{0}\right)^{1 / 2}
$$

The subscript 0 refers to current epoch values, and $\rho_{\Lambda}$ is the dark energy mass density. In the earliest times, $G$ is proportional to temperature, $T$, in both models, A, and B. We can furthermore show that, at inception,

$$
\begin{aligned}
& G / G_{0}=5.273 \times 10^{20} \\
& G / G_{0}=4.113 \times 10^{20}
\end{aligned}
$$

Both values are similar and quite large. We believe, nevertheless, that there could very well be a twenty order of magnitude increase in $G$ at the extremely high temperatures, indicated by Equations (5), and (6). We substitute Equations, (12), and, (13), into relation, (11). This gives

$$
\begin{aligned}
& \Lambda / \Lambda_{0}=2.78 \times 10^{41} \\
& \Lambda / \Lambda_{0}=1.69 \times 10^{41}
\end{aligned}
$$$$
\text { (model B) }
$$

We do not have the 121 order of magnitude difference between present and past values because we are stopping well short of the Planck temperature. Our inception temperature is of the order, $\approx 7 \times 10^{21} \mathrm{~K}$. See Equations, (5), and, (6). We have instead a more modest 41 order of magnitude increase as indicated by Equations, (14), and, (15).

With these models $\mathrm{A}$, and $\mathrm{B}$, it now appears, that in addition to deriving $w=-0.98$, as well as explaining (alleviating) the cosmological constant problem, we have a possible explanation for the younger age of the universe. The figure above gives an age which falls in line with the newly observed values. In fact, we found in the original reference, [4], that we had to modify the Hubble value towards lower values in order to conform to the 13.8 billion year estimate. If we insist on 13.8 billion year look-back time, then for model, A, we should chose $H_{0 A}=61.7 \mathrm{~km} /(\mathrm{s} \cdot \mathrm{Mpc})$. For model $\mathrm{B}$, the corresponding correction should be, $H_{0 B}=63.9 \mathrm{~km} /(\mathrm{s} \cdot \mathrm{Mpc})$. If, on the other hand, we stick to the $67.74 \mathrm{~km} /(\mathrm{s} \cdot \mathrm{Mpc})$ value, then we obtain the ages as indicated above in our figure /graph. This may provide the solution to the "tension" problem currently being discussed. We went into great detail in reference [4] to show that the time varying $G$ models can reproduce the $\Lambda \mathrm{CDM}$ model in all its essentials. It is only in the very early universe ( $a \leq 0.001$ ) that marked deviations occur between model, $\mathrm{A}$, or $\mathrm{B}$, versus the $\Lambda \mathrm{CDM}$ model. For most of cosmic evolution, our variable $G$ models give results similar to the predictions of the $\Lambda$ CDM model. In fact, in the limit where the quintessence parameter, $w$ approaches -1 , the time-varying $\dot{G} / G$ vanishes, and we are left with precisely the concordance model.

If $G$ is indeed varying with respect to cosmological time, then $G^{-1}$ must be replaced by a scalar field, as first suggested by Jordan [16]. In fact, we find that in this instance,

$$
\left\langle 0\left|\Phi^{2}\right| 0\right\rangle=G^{-1}=M_{G}^{2} /(\hbar c)
$$

here $M_{G}$ is the mass of the hypothetical massive particle representing gravity, 
which is changing with respect to cosmological time. We consider gravity, as exemplified by the coupling "constant", $G$, to be a property of the vacuum. The expression, Equation (16), can be compared to the magnetization in condensed matter physics, or the inverse Fermi constant, $G_{F}^{-1} \sim M_{W}^{2}$, in high energy physics. All have the same canonical mass dimension as, $G^{-1}$, defined above. We are close to $G^{-1}$ having achieved its full saturation value, as can be seen upon inspection of Equations, (7), and, (8), but we are not there yet. The mass is still evolving, albeit at a very slow rate in the present cosmological epoch. Our parametrizations indicate full saturation values at $a \approx 2$ and $a \approx 10$ for models, A, and $B$, respectively. In other words, full saturation is achieved when the universe will have doubled its present size for model, A. In model B for full saturation, the universe will have to increase its size by a factor of 10 .

We close by discussing a modified Planck scale. As is well known, the Planck length, the Planck mass, the Planck time, etc. are all given in terms of Newton's constant, and if Newton's constant varies, then, there is nothing fundamental about the Planck scale. The saturated values for $G$, however, indicated by Equations, (9), and (10), do not change. If there is anything comparable to Planck scale, then we should be using these values for a refined definition of Planck length, mass, time, etc. We therefore make the following adjustments,

$$
L_{P P}=\left(\hbar G_{\infty} / c^{3}\right)^{\frac{1}{2}}=\left(\hbar G_{0} / c^{3}\right)^{\frac{1}{2}}\left(G_{\infty} / G_{0}\right)^{\frac{1}{2}}=s L_{P}=s \times 1.62 \times 10^{-35} \mathrm{~m}
$$

The scale factor, $s$, can be found using Equations, (9), and (10). For model, A, we obtain $s_{A}=0.993$, whereas for model B, the corresponding value is,

$s_{B}=0.974$. In Equation, (17), $L_{P}=1.62 \times 10^{-35} \mathrm{~m}$, is the conventional Planck length, defined in terms of the present day value of Newton's constant, and $L_{P P}$ is the adjusted value using a saturated value for Newton's constant.

Similarly, one can show that,

$$
M_{P P}=\left(\hbar c / G_{\infty}\right)^{\frac{1}{2}}=\left(\hbar c / G_{0}\right)^{\frac{1}{2}}\left(G_{0} / G_{\infty}\right)^{\frac{1}{2}}=s^{-1} M_{P}=s^{-1} \times 2.18 \times 10^{-8} \mathrm{~kg}
$$

The quantity, $M_{P}=2.18 \times 10^{-8} \mathrm{~kg}$, is the conventional Planck mass, defined in terms of the present day value of Newton's constant, and $M_{P P}$ is the adjusted value, using a saturated value for Newton's constant. For models, $\mathrm{A}$, and B, the scale factor, $s^{-1}$ equals, $s_{A}^{-1}=1.007$, and, $s_{B}^{-1}=1.027$, respectively. The other Planck quantities can be adjusted correspondingly. We notice that because these scale factors are close to one, there is not much difference between the adjusted Planck scale and the conventional Planck scale. Nevertheless, there is a difference, if these models are to be taken seriously. An ultimate physical model/justification for the mathematical expressions, (1), and (2), remain to be explored and worked out.

\section{Conflicts of Interest}

The author declares no conflicts of interest regarding the publication of this paper. 


\section{References}

[1] Riess, A.G., Casertano, S., Yuan, W., Macri, L.M. and Scolnic, D. (2018) Large Magellanic Cloud Cepheid Standards Provide a 1\% Foundation for the Determination of the Hubble Constant and Stronger Evidence for Physics Beyond $\Lambda$ CDM. Astrophysical Journal, 861, 36. https://doi.org/10.3847/1538-4357/ab1422

[2] Ananthaswamy, A. (2019) Best-Yet Measurements Deepen Cosmological Crisis. Scientific American.

https://www.scientificamerican.com/article/best-yet-measurements-deepen-cosmol ogical-crisis

[3] Borenstein, S. New Study Says Universe Expanding Faster and Is Younger. https://apnews.com/fac50d45a19f4239848b1712cfd22c36

[4] Pilot, C. (2018) Is Quintessence an Indication of a Time-Varying Gravitational Constant? Journal of High Energy Physics, Gravitation and Cosmology, 5, 41-81. https://doi.org/10.4236/jhepgc.2019.51003 https://arxiv.org/abs/1803.06431

[5] Arnaud, M., et al. (2015) Planck 2015 Results. XIII. Cosmological Parameters. https://arXiv.org/abs/1502.1589v2

[6] Dirac, P.A.M. (1938) A New Basis for Cosmology. Proceedings of the Royal Society of London A, 165, 199-208. https://doi.org/10.1098/rspa.1938.0053

[7] Dirac, P.A.M. (1937) The Cosmological Constants. Nature, 139, 323. https://doi.org/10.1038/139323a0

[8] Dirac, P.A.M. (1974) Cosmological Models and the Large Numbers Hypothesis. Proceedings of the Royal Society of London A, 338, 439-446. https://doi.org/10.1098/rspa.1974.0095

[9] Mena Marugan, G.A. and Carneiro, S. (2002) Holography and the Large Number Hypothesis. Physical Review D, 65, Article ID: 087303. https://doi.org/10.1103/PhysRevD.65.087303

[10] Shao, C.-G., Shen, J., Wang, B. and Su, R.-K. (2006) Dirac Cosmology and the Acceleration of the Contemporary Universe. Classical and Quantum Gravity, 23, 3707-3720. https://doi.org/10.1088/0264-9381/23/11/003

[11] Ray, S., Mukhopadhyay, U. and Ghosh, P.P. (2007) Large Number Hypothesis: A Review.

[12] Unzicker, A. (2009) A Look at the Abandoned Contributions to Cosmology of Dirac, Sciama and Dicke. Annalen der Physik, 18, 57-70. https://doi.org/10.1002/andp.200810335

[13] Matthews, R. (1998) Dirac's Coincidences Sixty Years On. Astronomy \& Geophysics, 39, 619-620. https://academic.oup.com/astrogeo/article/39/6/6.19/203469 https://doi.org/10.1093/astrog/39.6.6.19

[14] Mather, J.C., et al. (1999) Calibrator Design for the COBE Far-Infrared Absolute Spectrophotometer. The Astrophysical Journal, 512, 511-520. https://doi.org/10.1086/306805

[15] Husdal, L. (2016) On Effective Degrees of Freedom in the Early Universe. Galaxies, 4,78 .

[16] Jordan, P. (1937) G Has to Be a Field. Naturwissenschaften, 25, 513. https://doi.org/10.1007/BF01498368 